\title{
Poor receptive joint attention skills are associated with atypical gray matter asymmetry in the posterior superior temporal gyrus of chimpanzees (Pan troglodytes)
}

\author{
William D. Hopkins ${ }^{1,2,3}$ *, Maria Misiura ${ }^{4}$, Lisa A. Reamer ${ }^{5}$, Jennifer A. Schaeffer ${ }^{2,3}$, Mary C. Mareno ${ }^{5}$ and $^{3}$ \\ Steven J. Schapiro ${ }^{5,6}$
}

\author{
${ }^{1}$ Neuroscience Institute, Georgia State University, Atlanta, GA, USA \\ 2 Language Research Center, Georgia State University, Atlanta, GA, USA \\ ${ }^{3}$ Division of Developmental and Cognitive Neuroscience, Yerkes National Primate Research Center, Atlanta, GA, USA \\ ${ }^{4}$ Department of Psychology, Agnes Scott College, Decatur, GA, USA \\ ${ }^{5}$ Department of Veterinary Sciences, The University of Texas MD Anderson Cancer Center, Bastrop, TX, USA \\ ${ }^{6}$ Department of Experimental Medicine, University of Copenhagen, Copenhagen, Denmark
}

\section{Edited by:}

Onur Gunturkun, Ruhr-University Bochum, Germany

\section{Reviewed by:}

Karen Lisa Bales, University of California Davis, USA

Alexis Garland, Ruhr University Bochum, Germany

\section{*Correspondence:}

William D. Hopkins, Neuroscience Institute and Language Research Center, Georgia State University, P.O. Box 5030, Atlanta, GA 30302, USA

e-mail:whopkins4@gsu.edu; whopkin@emory.edu

\begin{abstract}
Clinical and experimental data have implicated the posterior superior temporal gyrus as an important cortical region in the processing of socially relevant stimuli such as gaze following, eye direction, and head orientation. Gaze following and responding to different socio-communicative signals is an important and highly adaptive skill in primates, including humans. Here, we examined whether individual differences in responding to sociocommunicative cues was associated with variation in either gray matter (GM) volume and asymmetry in a sample of chimpanzees. Magnetic resonance image scans and behavioral data on receptive joint attention (RJA) was obtained from a sample of 191 chimpanzees. We found that chimpanzees that performed poorly on the RJA task had less GM in the right compared to left hemisphere in the posterior but not anterior superior temporal gyrus. We further found that middle-aged and elderly chimpanzee performed more poorly on the RJA task and had significantly less GM than young-adult and sub-adult chimpanzees. The results are consistent with previous studies implicating the posterior temporal gyrus in the processing of socially relevant information.
\end{abstract}

Keywords: joint attention, chimpanzees, superior temporal gyrus, brain asymmetry in cognition, brain development
At approximately 6-8 months of age, typically developing children begin to respond to a number of non-verbal sociocommunicative cues, including gaze, pointing and verbal bids (Adamson, 1996; Flom etal., 2006; Leavens, 2012). These are sometimes referred to as receptive joint attention (RJA) skills. Individual differences in RJA skill have been linked to the subsequent development of early linguistic skills, including comprehension and production of language, as well as other cognitive abilities, such as imitation learning and theory of mind (Mount etal., 1989; Charman etal., 2000; Slaughter and McConnell, 2003). For example, a number of studies have shown that the age of onset of both the initiation of, and response to, joint attention cues predicts the rate of language development in typically developing children (Bates et al., 1975, 1987; Carpenter etal., 1998; Morales etal., 2000; Nichols et al., 2005; Whalen etal., 2006; Mundy etal., 2007; Brooks and Meltzoff, 2008).

Not only is RJA a universal trait in typically developing children, there is also evidence for its existence in great apes and other primates, suggesting it has a long evolutionary history. Studies in a number of laboratories have shown that Old and New World monkeys and apes will not only follow gaze (Brauer et al., 2005; Rosati and Hare, 2009), but can follow gaze around barriers, and follow manual pointing gestures to specific locations
(Tomasello etal., 1999; Brauer et al., 2005; Amici et al., 2009). As with human infants (Moll and Tomasello, 2004), there are considerable individual differences in gaze following and RJA performance in nonhuman primates. For instance, Russell et al. (2011) examined, among a number of measures, gaze following on three trials in a sample of 83 chimpanzees. Fifteen percent of chimpanzees failed to follow gaze on all three trials, whereas $41 \%$ successfully followed gaze on all three trials. Herrmann et al. (2007, 2010) have reported similar individual differences in gaze following and comprehension of pointing responses in chimpanzees and bonobos.

Though the cognitive abilities of primates to respond to different socio-communicative cues are well documented, our understanding of the neural mechanisms underlying their expression are poorly understood. In the current study, we examined whether individual differences in RJA performance are linked to variation in the volume or asymmetry of the posterior superior temporal gyrus (p_STG) in chimpanzees. We focused on the p_STG as the cortical region of interest for several reasons. First, in Old World monkeys, single cell recording and reversible lesion studies have shown that neurons within the superior temporal gyrus and sulcus respond to certain social cues, such as eye gaze (Emery, 2000; Kamphius et al., 2009; Shepherd, 2010; Roy et al., 2012), and these results are consistent with 
fMRI findings in humans (Williams et al., 2005; Itier and Batty, 2009). Second, atypical patterns of asymmetry in the P_STG have been described in clinical populations in which deficits in social cognition and perception are prominent endophenotypes, notably schizophrenia (Barta et al., 1997; Klar, 1999; Kwon et al., 1999; Hirayasu et al., 2000; Sommer etal., 2001; Dollfus et al., 2005) and autism spectrum disorder (ASD; Zilbovicius et al., 2006; Jou etal., 2010; Chen etal., 2011). Third, in a recent review, Mundy and Newell (2007) proposed that responding to joint attention is associated with regions in the posterior superior temporal lobe and portions of the parietal lobe. For instance, in human adults, Williams etal. (2005) performed fMRI on subjects when they were engaged in joint attention compared to non-joint attention processing and found a significant number of brain regions active, including the ventromedial left prefrontal cortex (BA44, BA45), superior temporal gyrus (BA22), superior frontal cortex (BA10) anterior cingulate cortex (BA24), and regions within the basal ganglia (putamen and caudate). In terms of preverbal infants, far less is known, but studies employing scalp recording methods, such as EEG and ERPs, have reported significantly greater activity in posterior temporal and parietal regions when responding to joint attention cues (Mundy et al., 2000). These collective findings led us to focus on the p_STG as a targeted region potentially associated with RJA performance.

Chimpanzees are particularly valuable model species for understanding the neurobiology of social cognition for several reasons. First, as noted above, they have well developed RJA skills and, like humans, their responses to different sociocommunicative cues fall along a continuum. This study was designed to delineate several points on this continuum that might be useful for understanding human social cognition as it relates to different clinical population such as schizophrenia and ASD. Second, anatomically and cytoarchitectonically, there is considerable homology between the human and chimpanzee brain (Hopkins and Nir, 2010; Spocter et al., 2010; Hopkins, 2013). For instance, the sulcal landmarks used to quantify the planum temporale and planum parietale in humans and chimpanzees are nearly identical (Hopkins and Nir, 2010; Gilissen and Hopkins, 2013) and, like humans, chimpanzees show leftward asymmetries in these regions, which are not found in other nonhuman primate species (Gannon et al., 2008; Lyn et al., 2011).

To test the hypothesis of the role of p_STG in RJA proposed by Mundy and Newell (2007), we measured RJA skills in chimpanzees on a task developed by Dawson et al. (2002), previously employed with typically developing children, as well as those at risk for autism. We also quantified the gray matter (GM) volumes of the anterior and posterior, superior temporal gryus (STG) in these same chimpanzees. We hypothesized that if variation in RJA skills is associated with cortical organization within the STG, then significant differences would be found between chimpanzees that perform poorly compared to those who perform moderately or very well on this task. Based on previous results from structural and functional imaging studies, we further hypothesized that associations between GM volume and/or asymmetry would be specific to the posterior, but not anterior, region of the STG.

\section{MATERIALS AND METHODS SUBJECTS}

Subjects for this study included 191 captive chimpanzees (Pan troglodytes) housed at either The University of Texas MD Anderson Cancer Center (UTMDACC) or the Yerkes National Primate Research Center (YNPRC) of Emory University. There were 114 females and 77 males housed in social groups that ranged in size from 2 to 13 individuals. The chimpanzees ranged in age from 8 to 53 years $($ Mean $=26.24$, s.d. $=10.68)$. Based on the age range, we classified our chimpanzee sample into four age groups including sub-adult ( $8-16$ years), young-adult (17-25), middleaged (26-39 years) and elderly ( 40 years or older). Based on these cut points, there were 31 sub-adult, 75 young-adult, 55 middleaged, and 30 elderly chimpanzees in the sample. The age groups cut-points were adopted from previous studies in captive chimpanzees (Herndon, 2009; Lacreuse et al., 2014). Subjects had access to both indoor and outdoor enclosures throughout the day and night, and participation in the study task was voluntary. All procedures were approved by the local Institutional Animal Care and Use Committees and followed the Institute of Medicine guidelines for use of chimpanzees in research.

\section{PROCEDURE}

\section{Receptive joint attention}

The task used to measure RJA was identical to one developed by Dawson etal. (2002) in human children. Experiments were conducted with subjects either independent of their social group or divided into subgroups of two or three individuals, where the non-focal animals did not distract or interfere with the testing of the focal subject. Each subject received four test trials and a diagram of the trial procedure is shown in Figure 1. The goal of the task was to assess the number of social cues needed to elicit an orienting response from the subject. To accomplish this, each trial consisted of three hierarchical steps with an increasing number of social cues provided to the subjects in order to elicit an orienting response.

At the onset of testing for each trial, the focal chimpanzee would sit calmly in front of the experimenter they would engage them in some type of husbandry behavior. This might include the chimpanzee showing their foot, hand, arm or some other body part for inspection. When the chimpanzee was compliant with these requests, it was given small pieces of food. When the experimenter sensed that the chimpanzee was socially engaged with them, they would stop interacting with them and look over their head for $5 \mathrm{~s}$, then return to a neutral position and wait $5 \mathrm{~s}$ (Step 1). If the chimpanzee overtly oriented or looked back to where the experimenter had looked either during the cue or the 5-s following the trial was over and the subject was given a score of 1 . If the chimpanzee failed to look during the 10-s response window in Step 1, the experimenter would re-engage the chimpanzee in the husbandry-type activities again until she again felt as though the subject was socially engaged. At this point, the experimenter would look over the subject's head again and this time point with an extended arm/finger toward an imaginary object behind them for $5 \mathrm{~s}$ (Step 2). After this, the experimenter returned to her sitting position and waited an additional $5 \mathrm{~s}$ for the chimpanzee to respond. If the chimpanzee oriented or looked 


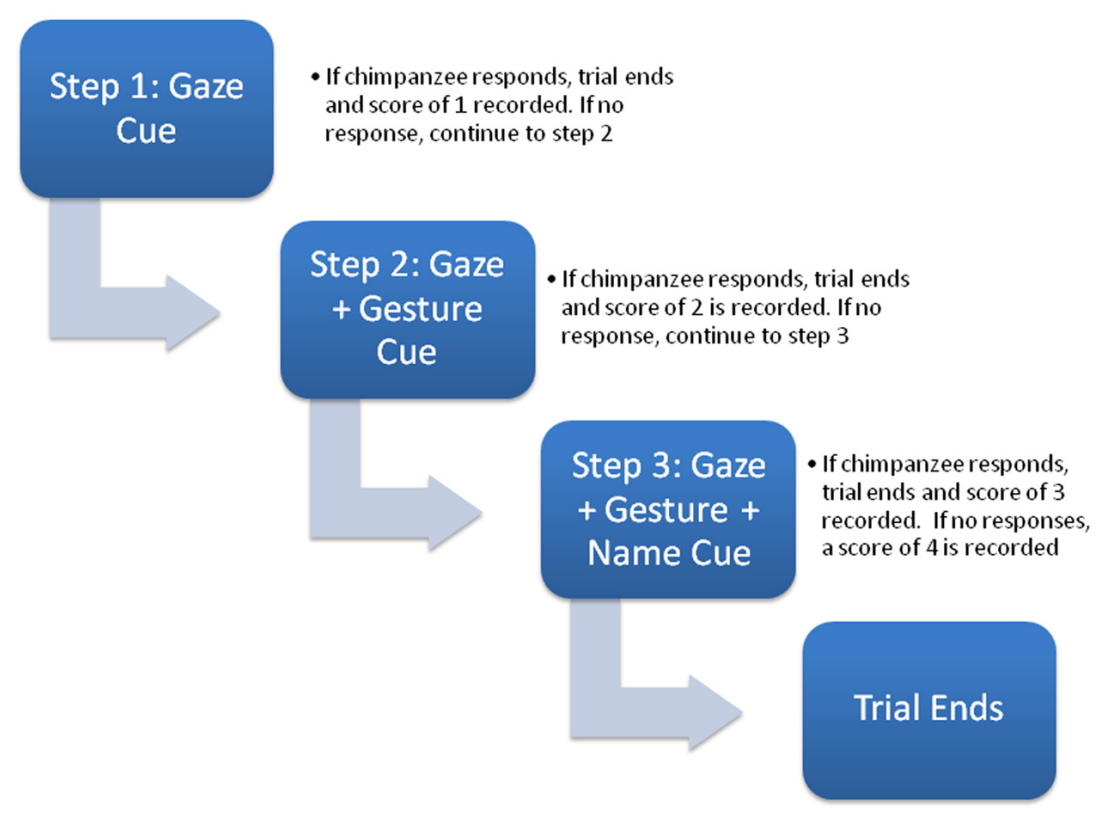

FIGURE 1 | Schematic diagram of the sequence of socio-communicative cues presented during each trial (see text for description).

back to where the experimenter had looked and pointed during the 10-s response window, the trial was over and the subject was given a score of 2 . If the focal chimpanzee failed to look during the response window in Step 2, as before, the experimenter re-engaged the chimpanzee in the husbandry-type activities. The experimenter, then again, looked over the subject's head, pointed with an extended arm/finger toward an imaginary object behind them and said the chimpanzee's name two times (Step 3). The experimenter then returned to her neutral sitting position and waited $5 \mathrm{~s}$ for the chimpanzees to respond. If the chimpanzee oriented or looked back to where the experimenter had indicated during the 10-s response window, the trial was over and the subject was given a score of 3 . If the chimpanzee failed to respond at the end of Step 3, it was given a score of 4 . To characterize the performance of the chimpanzees, we derived a composite overall score that reflected the average number of cues they needed to respond. For this variable, the score of each trial was summed across trials and divided by the number of trials (4; Mean_RJA). Higher Mean_RJA indicated that subjects needed, on average, more social cues to elicit an orienting response across all trials.

\section{MAGNETIC RESONANCE IMAGE COLLECTION}

All chimpanzees were scanned during their annual physical examination. Magnetic resonance image (MRI) scans followed standard procedures at the YNPRC and UTMDACC and were designed to minimize stress. Thus, the animals were first sedated with ketamine $(10 \mathrm{mg} / \mathrm{kg})$ or telazol $(3-5 \mathrm{mg} / \mathrm{kg})$ and were subsequently anesthetized with propofol (40-60 mg/kg/h). They were then transported to the MRI scanning facility and placed in a supine position in the scanner with their head in a human-head coil. Upon completion of the MRI, chimpanzees were briefly singly housed for 2-24 h to permit close monitoring and safe recovery from the anesthesia prior to return to their home social group. All procedures were approved by the Institutional Animal Care and Use Committees at YNPRC and UTMDACC and also followed the guidelines of the Institute of Medicine on the use of chimpanzees in research. Fifty-seven chimpanzees were scanned using a 3.0 Tesla scanner (Siemens Trio, Siemens Medical Solutions USA, Inc., Malvern, PA, USA). T1-weighted images were collected using a three-dimensional gradient echo sequence (pulse repetition $=2300 \mathrm{~ms}$, echo time $=4.4 \mathrm{~ms}$, number of signals averaged $=3$, matrix size $=320 \times 320$, with $0.6 \times 0.6 \times 0.6$ resolution). The remaining 134 chimpanzees were scanned using a 1.5T G.E. echo-speed Horizon LX MR scanner (GE Medical Systems, Milwaukee, WI, USA). T1-weighted images were collected in the transverse plane using a gradient echo protocol (pulse repetition $=19.0 \mathrm{~ms}$, echo time $=8.5 \mathrm{~ms}$, number of signals averaged $=8$, matrix size $=256 \times 256$, with $0.7 \times 0.7 \times 1.2$ resolution).

\section{REGION OF INTEREST}

Prior to quantification of the anterior (a_STG) and posterior superior temporal gyrus (p_STG), all T1-weighted MRI scans were realigned in the AC-PC plane, skull-stripped and segmented into GM, white matter and Cerebral spinal fluid following procedures that have been described in detail elsewhere (Zhang et al., 2001; Smith et al., 2004). The superior temporal gyrus (STG) was primarily quantified in the coronal plane but, when necessary, the landmarks could be viewed simultaneously in the axial or sagittal plane using ANALYZE 11.0 software. The superior border of the STG was the sylvian fissure; the inferior border was the superior temporal sulcus and the lateral border was the surface of the temporal lobe (see Figure 2). Beginning at the temporal pole in each 


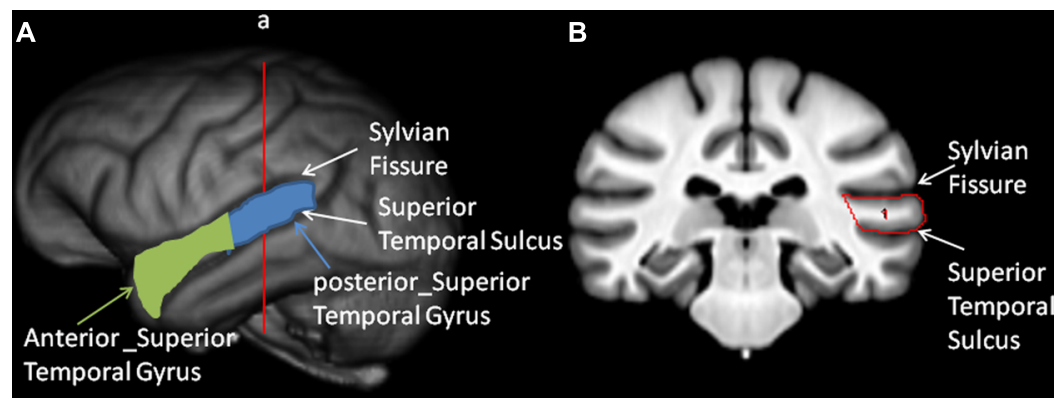

FIGURE 2 | Tracing of the anterior and posterior temporal lobe. Left panel: (A) 3-D reconstruction of the chimpanzee brain with the sylvian fissure (SF) and superior temporal sulcus (STS) labeled. Also, in green and light blue colors, the anterior and posterior superior temporal gyrus are outlined. Right panel: (B) Coronal view of the sulcl landmarks used to trace the superior temporal gyrus on a T-1 weighted MRI scan. hemisphere, an object map was drawn around the gyrus using the landmarks described above. Moving posteriorly in $1 \mathrm{~mm}$ increments, the object maps were drawn on each image and continued until the sylvian fissure or superior temporal sulcus terminated. In some cases, the posterior sylvian fissure bifurcated into an ascending and descending branch, and we always followed the descending ramus as the superior border of the STG. To divide the STG into anterior and posterior regions, the total length of the gyrus, which corresponded to the number of images on which an object map was drawn, was determined and the median slice was identified. Images lower or equal to the median were defined as the a_STG region and images higher than the median were defined as the p_STG. The median slice was typically found at or about the anterior location of Heschl's gyrus (HG). The object maps for each subject and hemisphere were saved. To calculate the GM volume of the a_STG and p_STG, the object maps that were traced on the T1-weighted scan for each hemisphere and region were applied to the segmented GM volume (see Figure 2B). The left and right hemisphere volumes $\left(\mathrm{mm}^{3}\right)$ were computed by summing all the voxels found within the a_STG and p_STG object maps. All the images were traced by a single individual (MM) and prior to data collection, intrarater agreement was established using intraclass correlation coefficients within a sample of 10 individual brains. Intraclass correlations were positive and significant for both the left $(r=0.922, p<0.01)$ and right $(r=0.972, p<0.05)$ hemispheres. The person ( $\mathrm{MM})$ tracing the brains was blind to the sex and individual performance of the chimpanzees on the RJA task.

\section{DATA ANALYSIS}

For each subject, we computed a percentage of GM volume by dividing the a_STG and p_STG GM values by the total GM volumes within each hemisphere. This was done to adjust for potential individual differences in total GM independent of the regions of interest. The percentage scores were averaged between the two hemispheres to create an overall estimate of GM for each region. In addition, we also computed asymmetry quotients (AQ) for GM within each region (GM_AQ_Ant, GM_AQ_Post). AQ scores were computed following the formula: $[\mathrm{AQ}=(\mathrm{R}-$ $\mathrm{L}) /((\mathrm{R}+\mathrm{L}) \times 5)]$ where $\mathrm{R}$ and $\mathrm{L}$ represent the respective $\mathrm{GM}$ percentages for the right and left hemispheres. Positive AQ values reflect right hemisphere biases and negative values reflect leftward asymmetries. The absolute value of the AQ indicates the strength or magnitude of the asymmetry. All analyses were performed using inferential statistics with alpha set to $p<0.05$. Post hoc analyses, when necessary, were conducted using Tukey's Honest Significant Difference test.

\section{RESULTS}

\section{RECEPTIVE JOINT ATTENTION}

In the initial analyses, we tested for sex and age effects on the Mean_RJA performance. For this analysis, we used analysis of variance with sex and age group as the between group factors, while the Mean_RJA scores were the dependent measure. We found a significant main effect for $\operatorname{sex} F(1,183)=4.288, p<0.04$ and a significant interaction between sex and age group $F(3,183)=4.364$, $p<0.006$. The mean Mean_RJA performance for males and females from each age group are shown in Table 1. Post hoc analysis indicated that elderly females did significantly worse than middleaged, young-adult and sub-adult females. For males, elderly and middle-aged individuals did significantly worse than young-adult and sub-adult apes.

\section{STG VOLUME AND ASYMMETRY}

We examined the effects of sex and age on STG volume and asymmetry. In the volumetric analysis, we used a mixed-model ANOVA with the standardized GM $z$-scores for the anterior and posterior STG serving as the repeated measure, while sex and age group were the between-group factors. This analysis revealed significant main effects for sex $F(1,183)=6.661, p<0.02$ and age $F(3,183)=2.837 p<0.04$. There was also a significant interaction between sex and temporal lobe region $F(1,183)=5.316, p<0.03$. For the age main effect, post hoc analysis indicated that elderly chimpanzees had smaller GM volumes compared to sub-adult and young adult, but not middle-aged chimpanzees. The mean percentage GM volumes in each group are shown in Table 1. For the interaction between sex and temporal lobe region, post hoc analysis indicated no significant difference in GM volume for the a_STG region; however, for the p_STG region, males (Mean $=2.27$, $\mathrm{SE}=0.054$ ) had relatively less GM than females (Mean $=2.56$, $\mathrm{SE}=0.054)$. No other significant main effects or interactions were found. 
Table 1 | Average Mean_RJA and percentage GM volumes (+SE) for male and female chimpanzees in each age group.

\begin{tabular}{lllll}
\hline & \multicolumn{4}{c}{ Age groups } \\
\cline { 2 - 5 } & Sub-adult & Young-adult & Middle-aged & Elderly \\
\hline Mean_RJA & & & 1.81 & 2.63 \\
Females & 1.95 & 1.99 & $(0.14)$ & $(0.18)$ \\
& $(0.19)$ & $(0.14)$ & 2.85 & 2.33 \\
Males & 2.21 & 2.13 & $(0.20)$ & $(0.28)$ \\
& $(0.23)$ & $(0.14)$ & 2.33 & 2.48 \\
Overall & 2.08 & 2.06 & $(0.12)$ & $(0.16)$ \\
& $(0.15)$ & $(0.09)$ & & \\
Percentage & GM volume & & 2.56 & 2.32 \\
Females & 2.68 & 2.58 & $(0.06)$ & $(0.09)$ \\
& $(0.11)$ & $(0.05)$ & 2.28 & 2.19 \\
Males & 2.55 & 2.34 & $(0.11)$ & $(0.15)$ \\
& $(0.13)$ & $(0.07)$ & 2.42 & 2.26 \\
Overall & 2.62 & 2.46 & $(0.07)$ & $(0.09)$ \\
& $(0.08)$ & $(0.05)$ & & \\
\hline
\end{tabular}

For asymmetries in the a_STG and P_STG, we also used a mixed model ANOVA with the AQ scores for each region serving as the repeated measure while sex and age group were the between group factors. A significant main effect for region was found $F(1,183)=27.624, p<0.001$. The mean AQ scores for the p_STG region $($ Mean $=-0.080, \mathrm{SE}=0.013)$ were more leftward than the a_STG region (Mean $=0.023, \mathrm{SE}=0.013)$. Indeed, one sample $t$ tests on the AQ scores revealed a significant populationlevel leftward bias for the p_STG $t(190)=-7.214, p<0.001$, but no significant bias for the a_STG region $t(190)=0.709, p=0.479$.

\section{RELATIONSHIP BETWEEN MEAN_RJA AND STG VOLUME AND ASYMMETRY}

In the next set of analyses, we integrated the measures of GM volume and asymmetry for the a_STG and P_STG regions into a series of partial correlation analyses as a means of predicting individual differences in RJA performance. Because we previously showed that age and sex influenced RJA performance, we sought to determine whether variation in either GM volume or asymmetry would account for a significant proportion of variability in performance over and above that of the variables of sex and age. Thus, we performed partial correlation coefficients between Mean_RJA performance and the a_STG and P_STG standardized GM volumes and AQ scores. The only significant partial $r$-value was between Mean_RJA performance and p_STG AQ scores (beta $=0.155$, $p<0.04)$. Subjects with more rightward AQ scores showed poorer RJA performance.

\section{RELATIONSHIP BETWEEN GAZE PERFORMANCE ALONE AND STG VOLUME AND ASYMMETRY}

The previous analyses focused on the association between Mean_RJA performance and variation in GM volume and asymmetry in the a_STG and P_STG regions. Because gaze following and response to gaze cues alone are important factors linked to variation in P_STG organization, we further examined whether performance on the gaze-following cue alone was associated with the neuroanatomical measures. For this analysis, we computed the number of trials on which the chimpanzees responded to the gaze cue alone. Scores could range from 0 to 4 (a 4 was recorded when the subject responded to gaze alone on all four trials). Based on these data, and in order to increase statistical power, we classified the chimpanzees into one of three groups, including poorer than average (score $=0$, PTA_Gaze), average $($ score $=1$ or 2,AVG_Gaze) or better than average (score of a 3 or 4 , BTA_Gaze). We then compared the a_STG and p_STG volume and asymmetry scores between these groups as well as between sexes and age groups using analysis of variance. No significant main effects or interactions were found between gaze performance, sex and the a_STG and P_STG GM volume measures; however, for the AQ scores, we found a significant main effect for gaze performance on the p_STG scores $F(2,167)=4.054, p<0.02$. The mean p_STG AQ scores for the BTA_Gaze, AVG_Gaze, PTA_Gaze groups are shown in Figure 3. Post hoc analysis indicated that the mean P_STG AQ scores were significantly more leftward for the BTA_Gaze group compared to the PTA_Gaze group but did not differ from the AVG_Gaze group. No other significant differences were found. For the a_STG AQ scores, no significant main effects or interactions were found.

\section{DISCUSSION}

The results of this study reveal several important findings. First, poorer performance on a task designed to assess RJA is associated with greater rightward asymmetries in the posterior, but not anterior, portion of the superior temporal gyrus. Second, we found significant age-related changes in performance on the RJA task and overall GM volume within the superior temporal gyrus. For the RJA task, older subjects performed more poorly than younger subjects. Further, the onset in decline on performance started at a younger age in males compared to females. For the GM volume, older subjects had lower percentages of GM compared to younger individuals.

With regard to the association between RJA and gaze performance and atypical asymmetries in the P_STG, our findings in chimpanzees are consistent with the hypothesis proposed by Mundy and Newell (2007), and are in general agreement with results in human clinical populations in which deficits in sociocommunicative abilities are a significant endophenotype, such as schizophrenia (Sommer et al., 2001) or ASD (Boddaert et al., 2004; Zilbovicius et al., 2006). To be clear, we are not suggesting that our chimpanzees that respond poorly to socio-communicative cues are schizophrenic or autistic but rather that individual variation in RJA performance appears to be explicitly linked to asymmetries in the p_STG, but not the a_STG. We emphasize the word atypical asymmetry in this discussion because it is important to emphasize that the chimpanzees, as a group, show a leftward asymmetry in the GM volume of p_STG. Thus, individuals who fail to show a bias, or those with reversed asymmetries in the p_STg, are the ones who perform poorly on the RJA task.

The finding of a significant association between RJA task performance and atypical asymmetries in the P_STG also bears 


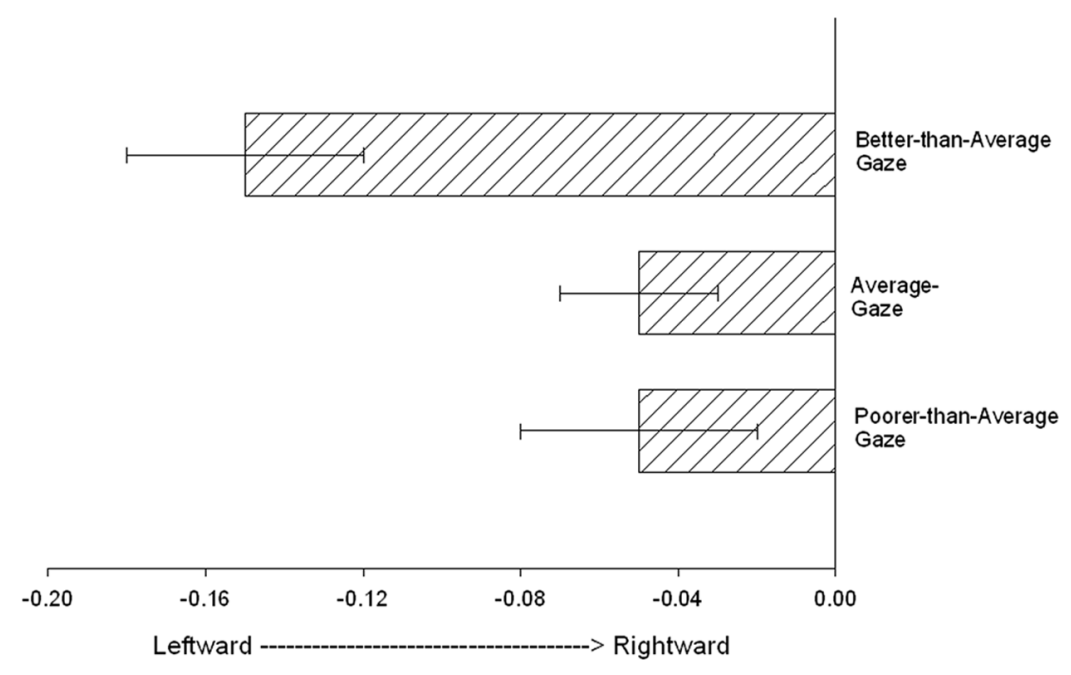

FIGURE 3 |The mean posterior superior temporal gyrus (p_STG) AQ scores (+/- standard error) for the Better-than-average_Gaze, Average_Gaze, Poorer-than-average_Gaze groups.

directly on theoretical and applied views of the role of brain asymmetries on individual fitness. A number of researchers have argued that having an asymmetrical brain confers some advantages from an evolutionary perspective (Ghirlanda and Vallortigara, 2004; Vallortigara and Rogers, 2005). With the context of the results reported here, it might be suggested that having an asymmetrical p_STG (and indeed, a leftward asymmetry) provides individuals with increased sensitivity for monitoring socio-communicative cues from conspecifics, such as gaze direction, head orientation and gestures. Many of these cues would be potentially important for selecting mates and/or avoiding conflict and agonistic encounters with conspecifics, and therefore afford some advantages to those individuals.

We also found age-related changes in both RJA performance and standardized GM volume. With respect to RJA, older chimpanzees performed more poorly than younger individuals. Similarly, older individuals had lower standardized GM volumes than younger individuals (see Figure 2). There is very little data on age-related changes in cognition and cortical organization in chimpanzees, but the findings reported here are partially consistent with existing data, though they also differ in some important ways. Recently, in a sample of 36 female chimpanzees, Lacreuse et al. (2014) reported age-related changes in response to gaze following, with older subjects performing more poorly. The results reported here are largely consistent with this finding, though in a much larger sample of chimpanzees that also included males. The inclusion of males was relevant in the present study because the findings showed that the decline in RJA performance occurred at an earlier age in males than it did in females. Life history and survival tables for chimpanzees have shown pronounced sex differences in life span, with males dying, on average, 7 years earlier than females (Dyke etal., 1995; Hill et al., 2001). Thus, the early decline in RJA performance abilities in males compared to females is consistent with the differences in relative life span and mortality between the sexes.

The evidence for age-related decline in the GM volume within the temporal lobe is, as far as we know, the first compelling evidence of age-related decline in cortical organization in chimpanzees. Several studies in chimpanzees that have examined age-related decline in total brain volume and weight, white matter volume, frontal lobe gray and white matter volume and hippocampal volume have failed to find age-related changes (Herndon et al., 1999; Sherwood etal., 2011; Chen et al., 2013). Therefore, the significant effect of age on GM volume was not anticipated and certainly contradicts previous findings in chimpanzees. However, the data presented here differ from these previous studies in two important ways that might explain the discrepancy in findings. First, we had a much larger sample size than previous studies (previous largest sample size was $n=97$ ), particularly among males and individuals within the elderly group. Second, we focused on the temporal lobe GM in this study, a region that has not, until now, been explicitly quantified in previous studies examining age-related changes in cortical organization in chimpanzees.

In summary, individual differences in RJA performance was associated with in GM asymmetries in the p_STG in chimpanzees. These findings are consistent with evidence of the role of the posterior superior temporal lobe in the processing of socially relevant information in humans and monkeys. What factors or mechanisms underlie both variation in RJA performance and p_STG asymmetries are not clear from this study, but the findings indicate that additional consideration and investigation are warranted. We would further add that this study focused only on anatomy, but examining the functional role of the P_STG in relation to RJA performance should be explored in future studies as a means of understanding the ontogenetic and phylogenetic factors that underlie the perception of socially relevant communicative cues in primates, including humans. 


\section{ACKNOWLEDGMENTS}

This research was supported by NIH grants MH-92923, NS-42867, NS-73134, HD-56232 and HD-60563, and Cooperative Agreement U42-OD011197. American Psychological Association and Institute of Medicine guidelines for the treatment of animals were followed during all aspects of this study. We would like to thank Yerkes National Primate Research Center and The University of Texas MD Anderson Cancer Center and their respective veterinary and animal care staffs for assistance in MR imaging.

\section{REFERENCES}

Adamson, L. R. (1996). Communication Development During Infancy. Boulder, CO: Westview.

Amici, F., Aureli, F., Visalberghi, E., and Call, J. (2009). Spider monkeys (Ateles geoffroyi) and capuchin monkeys (Cebus apella) follow gaze around barriers: evidence for perspective taking? J. Comp. Psychol. 123, 368-374. doi: 10.1037/a00 17079

Barta, P., Pearlson, G., Brill, L. B., Royall, R., McGilchrist, I. K., Pulver, A. E., et al. (1997). Planum temporale asymmetry reversal in schizophrenia: replication and relationship to gray matter abnormalities. Am. J. Psychiatry 154, 661-667.

Bates, E., Camaioni, L., and Volterra, V. (1975). Performatives prior to speech. Merrill-Palmer Q. 21, 205-226.

Bates, E., O’Connell, B., and Shore, C. (1987). "Language and communication in infancy," in Handbook of Infant Development, ed. J. Osofsky (New York: Wiley), 149-203.

Boddaert, N., Chabane, N., Gervais, H., Good, C. D., Bourgeois, M., Plumet, M.-H., et al. (2004). Superior temporal sulcus anatomical abnormalities in childhood autism: a voxel-based morphometry MRI study. NeuroImage 23, 364-369. doi: 10.1016/j.neuroimage.2004.06.016

Brauer, J., Call, J., and Tomasello, M. (2005). All great ape species follow gaze to distant locations and around barriers. J. Comp. Psychol. 119, 145-154. doi 10.1037/0735-7036.119.2.145

Brooks, R., and Meltzoff, A. N. (2008). Infant gaze following and pointing predict accelerated vocabulary growth through two years of age: a longitudinal, growth curve modeling study. J. Child Lang. 35, 207-220. doi: 10.1017/S030500090700829X

Carpenter, M., Nagell, K., Tomasello, M., Butterworth, G., and Moore, C. (1998) Social cognition, joint attention, and communicative competence from 9 to 15 months of age. Monogr. Soc. Res. Child Dev. 63, i-vi, 1-143.

Charman, T., Baron-Cohen, S., Swettenham, J., Baird, G., Cox, A., and Drew, A. (2000). Testing joint attention, imitation and play as infancy precursors to language and theory of mind. Cogn. Dev. 15, 481-498. doi: 10.1016/S08852014(01)00037-5

Chen, R., Jiao, Y., and Hersovits, E. H. (2011). Structural MRI in autism spectrum disorder. Pediatr. Res. 69, 63R-68R. doi: 10.1203/PDR.0b013e318212c2b3

Chen, X., Errangi, B., Li, L., Glasser, M. F., Westyle, L. T., Fjell, A. M., et al. (2013). Brain aging in humans, chimpanzees (Pan troglodytes) and hresus macaques (Macaca mulatta): Magnetic resonance images of macro- and microstructural changes. Neurobiol. Aging 34, 2248-2260. doi: 10.1016/j.neurobiolaging.2013. 03.028

Dawson, G., Munson, J., Estes, A., Osterling, J., McPartland, J., Toth, K., et al. (2002). Neurocognitive function and joint attention ability in young children with autism spectrum disorder versus developmental delay. Child Dev. 73, 345-358 doi: 10.1111/1467-8624.00411

Dollfus, S., Razafimandimby, A., Delamillieure, P., Brazo, P., Joliot, M., Mazoyer, B., et al. (2005). Atypical Hemispheric Specialization for language in right-handed schizophrenia Patients. Biol. Psychiatry 57, 1020-1028. doi: 10.1016/j.biopsych.2005.01.009

Dyke, B., Williams-Blangero, S., Mamelka, P. M., and Goodwin, W. J. (1995). Future costs of chimpanzees in U.S. research facilities. ILAR J. 37, 193-198.

Emery, N. J. (2000). The eyes have it: the neuroethology, function and evolution of social gaze. Neurosci. Biobehav. Rev. 24, 581-604. doi: 10.1016/S01497634(00)00025-7

Flom, R., Lee, K.S., and Muir, D. (eds). (2006). Gaze-following: Its Development and Significance. London: Psychology Press.
Gannon, P. J., Kheck, N., and Hof, P. R. (2008). Leftward interhemispheric asymmetry of macaque monkey temporal lobe language area homolog is evident at the cytoarchitectural, but not gross anatomic level. Brain Res. 1199, 62-73. doi: 10.1016/j.brainres.2007.12.041

Ghirlanda, S., and Vallortigara, G. (2004). The evolution of brain lateralization: a game-theoretical analysis of population structure. Proc. R. Soc. Lond. 271, 853-857. doi: 10.1098/rspb.2003.2669

Gilissen, E., and Hopkins, W. (D). (2013). Asymmetries in the parietal operculum in chimpanzees (Pan troglodytes) in relation to handedness for tool use. Cereb Cortex 23, 411-422. doi: 10.1093/cercor/bhs029

Herndon, J. G. (2009). The grandmother effect: implications for studies on aging and cognition. Gerontlogy 56, 73-79. doi: 10.1159/000236045

Herndon, J. G., Tigges, J., Anderson, D. C., Klumpp, S. A., and McClure, H. M. (1999). Brain weight throughout the life span of the chimpanzee. J. Comp. Neurol. 409, 567-572. doi: 10.1002/(SICI)1096-9861(19990712)409:4<567::AID$\mathrm{CNE} 4>3.0 . \mathrm{CO} ; 2-\mathrm{J}$

Herrmann, E., Call, J., Hernandez-Lloreda, M. V., Hare, B., and Tomasello, M. (2007). Humans have evolved specialized skills of social cognition: the cultural intelligence hypothesis. Science 317, 1360-1366. doi: 10.1126/science. 1146282

Herrmann, E., Hare, B., Call, J., and Tomasello, M. (2010). Differences in the cognitive skills of bonobos and chimpanzees. PLoS ONE 5:e12438. doi: 10.1371/journal.pone.0012438

Hill, K., Boesch, C., Goodall, J., Pusey, A. E., Williams, J., and Wrangham, R. (2001). Mortality rates among wild chimpanzees. J. Hum. Evol. 40, 437-450. doi: 10.1006/jhev.2001.0469

Hirayasu, Y., McCarley, R. W., Salisbury, D. F., Tanaka, S., Kwon, J. S., Frumin, M., et al. (2000). Planum temporale and Heschl gyrus volume reduction in schizophrenia. Arch. Gen. Psychiatry 57, 692-699. doi: 10.1001/archpsyc.57.7.692

Hopkins, W. D. (2013). Behavioral and brain asymmetries in chimpanzees: a case for continuity. Ann. N. Y. Acad. Sci. 1288, 27-35.

Hopkins, W. D., and Nir, T. (2010). Planum temporale surface area and grey matter asymmetries in chimpanzees (Pan troglodytes): The effect of handedness and comparison within findings in humans. Behav. Brain Res. 208, 436-443. doi: 10.1016/j.bbr.2009.12.012

Itier, R. J., and Batty, M. (2009). Neural bases of eye and gaze processing: the core of social cognition. Neurosci. Biobehav. Rev. 33, 843-863. doi 10.1016/j.neubiorev.2009.02.004

Jou, R. J., Minshew, N. J., Keshavan, M. S., Vitale, M. P., and Hardan, A. Y. (2010). Enlarged right superior temporal gyrusin children and adolescents with autism. Brain Res. 1360, 205-212. doi: 10.1016/j.brainres.2010.09.005

Kamphius, S., Dicke, P. W., and Thier, P. (2009). Neuronal substrates for gaze following in monkeys. Eur. J. Neurosci. 29, 1732-1738. doi: 10.1111/j.14609568.2009.06730.x

Klar, A. J. (1999). Genetic models of handedness, brain lateralization, schizophrenia, and manic-depression. Schizophr. Res. 39, 207-218. doi: 10.1016/S09209964(99)00075-4

Kwon, J. S., McCarley, R. W., Hirayasu, Y., Anderson, J. E., Fischer, I. A., Kikinis, R., et al. (1999). Left planum temporale reduction in schizophrenia. Arch. Gen. Psychiatry 56, 142-148. doi: 10.1001/archpsyc.56.2.142

Lacreuse, A., Russell, J. L., Hopkins, W. D., and Herndon, J. G. (2014). Cognitive and motor aging in female chimpanzees. Neurobiol. Aging 35, 623-632. doi 10.1016/j.neurobiolaging.2013.08.036

Leavens, D.A. (2012). "Joint attention: twelve myths," in Joint Attention: New developments in Pyschology, Philosophy of Mind and Social Neuroscience, ed. A. Seemann (Cambridge, MA: MIT Press), 43-72.

Lyn, H. L., Pierre, P., Bennett, A. J., Fears, S. C., Woods, R. P., and Hopkins, W. D. (2011). Planum temporale grey matter asymmetries in chimpanzees (Pan troglodytes), vervet (Chlorocebus aethiops sabaeus), rhesus (Macaca mulatta) and bonnet (Macaca radiata) monkeys. Neuropsychologia 49, 2004-2012. doi: 10.1016/j.neuropsychologia.2011.03.030

Moll, H., and Tomasello, M. (2004). 12- and 18-month-old infants follow gaze to spaces behind barriers. Dev. Sci. 7, F1-F9. doi: 10.1111/j.1467-7687.2004.00315.x

Morales, M., Mundy, P., Delgado, C. E. F., Yale, M., Messinger, D., Neal, R., et al. (2000). Responding to joint attention across the 6-through 24-month age period and early language acquisition. J. Appl. Dev. Psychol. 21, 283-298. doi: 10.1016/S0193-3973(99)00040-4 
Mount, R., Reznick, S. J., Kagan, J., Hiatt, S., and Szpak, M. (1989). Direction of gaze and emergence of speech in the second year. Brain Lang. 36, 406-410. doi: 10.1016/0093-934X(89)90076-X

Mundy, P., Block, J., Delgado, C., Pomares, Y., Van Hecke, A. V., and Parlade, M. V. (2007). Individual differences and the development of joint attention in infancy. Child Dev. 78, 938-954. doi: 10.1111/j.1467-8624.2007.01042.x

Mundy, P., Card, J., and Fox, N. (2000). EEG correlates of the development of infant joint attention skills. Dev. Psychobiol. 36, 325-338. doi: 10.1002/(SICI)10982302(200005)36:4<325::AID-DEV7>3.0.CO;2-F

Mundy, P., and Newell, L. (2007). Attention, joint attention, and social cognition. Curr. Dir. Psychol. Sci. 16, 269-274. doi: 10.1111/j.1467-8721.2007.00518.x

Nichols, K. E., Fox, N. A., and Mundy, P. (2005). Joint attention, selfrecognition and neurocognitive function in toddlers. Infancy 7, 35-51. doi: 10.1207/s15327078in0701_4

Rosati, A. G., and Hare, B. (2009). Looking past the model species: diversity in gaze-following skills in primates. Curr. Opin. Neurobiol. 19, 45-51. doi: 10.1016/j.conb.2009.03.002

Roy, A., Shepard, S. V., and Platt, M. L. (2012). Reversible inactivation of pSTS supresses social gaze following in the macaque (Macaca mulatta). Soc. Cogn. Affect Neurosci. [Epub ahead of print]. doi: 10.1093/scan/nss123

Russell, J. L., Lyn, H., Schaeffer, J. A., and Hopkins, W. D. (2011). The role of sociocommunicative rearing environments in the development of social and physical cognition in apes. Dev. Sci. 14, 1459-1470. doi: 10.1111/j.1467-7687.2011.01090.x

Shepherd, S. (2010). Following gaze: gaze-following behavior as a window to social cognition. Front. Integr. Neurosci. 4:5. doi: 10.3389/fnint.2010.00005

Sherwood, C. C., Gordon, A. D., Allen, J. S., Phillips, K. A., Erwin, J. M., Hof, P. R., et al. (2011). Aging of the cerebral cortex differs between humans and chimpanzees. Proc. Natl. Acad. Sci. U.S.A. 108, 13029-13034. doi: 10.1073/pnas.1016709108

Slaughter, V., and McConnell, D. (2003). Emergence of joint attention: relationships between gaze following, social referencing, imitation and naming in infancy. $J$. Genet. Psychol. 164, 54-71. doi: 10.1080/00221320309597503

Smith, S. M., Jenkinson, M., Woolrich, M. W., Beckmann, C. F., Behrens, T. E. J., Johansen-Berg, H., et al. (2004). Advances in functional and structural MR image analysis and implementation of FSL. NeuroImage 23, 208-219. doi: 10.1016/j.neuroimage.2004.07.051

Sommer, I., Ramsey, N., and Kahn, R. (2001). Handedness, language lateralisation and anatomical asymmetry in schizophrenia: meta-analysis. Br. J. Psychiatry 178, 344-351. doi: 10.1192/bjp.178.4.344

Spocter, M. A., Hopkins, W. D., Garrison, A. R., Stimpson, C. D., Erwin, J. M., Hof, P. R., et al. (2010). Wernicke's area homolog in chimpanzees (Pan troglodytes): probabilstic mapping, asymmetry and comparison with humans. Proc. Biol. Sci. 277, 2165-2174. doi: 10.1098/rspb.2010.0011

Tomasello, M., Hare, B., and Agnetta, B. (1999). Chimpanzees, Pan troglodytes, follow gaze direction geometrically. Animal Behav. 58, 769-777. doi: 10.1006/anbe.1999.1192

Vallortigara, G., and Rogers, L. J. (2005). Survival with an asymmetrical brain: advantages and disadvantages of cerebral lateralization. Behav. Brain Sci. 28, 574. doi: 10.1017/S0140525X05000105

Whalen, C., Schreibman, L., and Ingersoll, B. (2006). The collateral effect of joint attention training on social initiations, positive affect, imitation, and spontaneous speech in young children with autism. J. Autism. Dev. Disord. 36, 655-664. doi: 10.1007/s10803-0060108-Z

Williams, H. G., Waiter, G. D., Perra, O., Perrett, D. I., and Whiten, A. (2005). A fMRI study of joint attention. NeuroImage 25, 133-140. doi: 10.1016/j.neuroimage.2004.10.047

Zhang, Y., Brady, M., and Smith, S. M. (2001). Segmentation of the brain MR images through hidden Markov random filed model and expectation-maximization algorithm. IEEE Trans. Med. Imaging 20, 45-57. doi: 10.1109/42.906424

Zilbovicius, M., Meresse, I., Chabane, N., Brunelle, F., Samson, Y., and Boddaert, N. (2006). Autism, the superior temporal sulcus and social perception. Trends Neurosci. 29, 359-366. doi: 10.1016/j.tins.2006.06.004

Conflict of Interest Statement: The authors declare that the research was conducted in the absence of any commercial or financial relationships that could be construed as a potential conflict of interest.

Received: 21 October 2013; paper pending published: 08 December 2013; accepted: 05 January 2014; published online: 29 January 2014.

Citation: Hopkins WD, Misiura M, Reamer LA, Schaeffer JA, Mareno MC and Schapiro SJ (2014) Poor receptive joint attention skills are associated with atypical gray matter asymmetry in the posterior superior temporal gyrus of chimpanzees (Pan troglodytes). Front. Psychol. 5:7. doi: 10.3389/fpsyg.2014.00007

This article was submitted to Cognition, a section of the journal Frontiers in Psychology. Copyright (C) 2014 Hopkins, Misiura, Reamer, Schaeffer, Mareno and Schapiro. This is an open-access article distributed under the terms of the Creative Commons Attribution License (CC BY). The use, distribution or reproduction in other forums is permitted, provided the original author(s) or licensor are credited and that the original publication in this journal is cited, in accordance with accepted academic practice. No use, distribution or reproduction is permitted which does not comply with these terms. 